time $(\beta=-0.50, \mathrm{p}<0.001)$ and procedure duration $(\beta=-$ $0.26, p=0.002$ ) were associated with procedure number. Time for sheath insertion, fluoroscopy time, radiation dose, and contrast volume were not associated with procedure number. Segmented linear regression identified break-points of 33 for sheath to first vessel time and 11 for procedure duration, which corresponded to the procedure number after which these outcomes trended down. LOESS models for time to sheath placement, procedure duration, fluoroscopy time, and radiation dose predicted minimum values between procedure numbers 40 - 50 .

Conclusion Transitioning from pTRA to dTRA for diagnostic cerebral angiography is feasible and safe. The learning curve is overcome between procedures 11 and 33, while further refinement in performance occurs through procedures 40 - 50 .

Disclosures H. Hoffman: None. K. Bunch: None. T. Mikhailova: None. J. Cote: None. A. Ashok Kumar: None. H. Masoud: None. G. Gould: None.

\section{E-100 ENDOVASCULAR MANAGEMENT OF REFRACTORY NASOPHARYNGEAL AND OROPHARYNGEAL BLEEDING: CASE SERIES}

H Hoffman*, G Gould. SUNY Upstate Medical University, Syracuse, NY

\subsection{6/neurintsurg-2021-SNIS.195}

Introduction Patients with oronasal bleeding refractory to packing or cautery may be referred for endovascular embolization. Little data is available regarding the safety and efficacy of endovascular treatment in this setting. We sought to characterize this by retrospectively analyzing our institutional experience.

Methods Consecutive endovascular procedures for nasopharyngeal or oropharyngeal bleeding performed at a single institution were reviewed. Outcomes included immediate procedural success, delayed rebleeding requiring additional surgical or endovascular treatment, procedural complications, and mortality occurring during the index hospitalization. Binary logistic regression was used to identify factors associated with outcomes.

Results A total of 44 interventions performed on 38 patients were included. The mean $( \pm \mathrm{SD})$ age was 60.39 years \pm 15.79 and the majority $(77.3 \%)$ of patients were male. Of the 38 patients, $21(55.3 \%)$ had squamous cell carcinoma of the head and neck and $10(26.3 \%)$ were hemodynamically unstable preoperatively. Coils were used in $23(52.3 \%)$ cases, particles were used in 18 (40.9\%) cases, and Onyx was used in $9(20.5 \%)$ cases. Immediate cessation of bleeding was achieved in $42(95.5 \%)$ cases, but delayed rebleeding occurred in $8(18.2 \%)$ cases at a median of 1 day (range 0 - 9) after the index procedure. In the binary logistic regression, preoperative hypotension (OR 5.73, 95\% CI 1.05 - 37.95) and use of coils (OR 8.61, 95\% CI 1.22 178.1) were associated with delayed rebleeding. None of the other embolic materials were associated with rebleeding. Procedural complications included stroke in 1 patient. Mortality occurred in $5(13.2 \%)$ patients but was related to the patient's underlying cancer burden in 4 . In the binary logistic regression, preoperative hypotension was independently associated with mortality (OR 19.23, 95\% CI 2.16 449.79).
Conclusion Endovascular treatment of oronasal bleeding is safe and effective at achieving hemostasis but associated with an $18.2 \%$ rebleeding rate.

Disclosures H. Hoffman: None. G. Gould: None.

\section{E-101 BALLOON-ASSISTED ROADMAP TECHNIQUE TO ENABLE SUCCESSFUL FLOW DIVERSION AND ADJUVANT COILING OF A HIGH-FLOW DIRECT CAROTID- CAVERNOUS FISTULA}

F Jareczek*, V Padmanaban, D Wilkinson. Neurosurgery, Penn State Health Milton S Hershey Medical Center, Hershey, PA

\subsection{6/neurintsurg-2021-SNIS. 196}

Use of flow diverters as a first-line treatment for direct carotid cavernous fistula (CCF) is a relatively new approach in the neurointerventional field. Safe and successful deployment of a flow diverter requires adequate imaging of the parent vessel distal to the location of the fistulous connection, which may be challenging in the setting of high-flow CCF. Temporary balloon occlusion of the fistulous connection is a novel means of facilitating acquisition of the necessary images for producing a roadmap to facilitate flow diverter deployment. Here we present the case of a traumatic high-flow direct CCF successfully treated with flow diverter-assisted coil embolization using a roadmap acquired with temporary balloon occlusion of the fistulous connection.

Disclosures F. Jareczek: None. V. Padmanaban: None. D. Wilkinson: None.

\section{E-102 TRANSPORT DISTANCES FOR BYPASS AND NON- BYPASS ROUTING MODELS OF STROKE PATIENTS IN THE UNITED STATES}

${ }^{1} \mathrm{C} \mathrm{Yu}^{*},{ }^{1} \mathrm{P}$ Panagos, ${ }^{1,2} \mathrm{~A}$ Kansagra. 'Washington University School of Medicine, St. Louis, MO; ${ }^{2}$ Mallinckrodt Institute of Radiology, St. Louis, MO

\subsection{6/neurintsurg-2021-SNIS.197}

Background and Purpose Acute interventions for ischemic stroke include intravenous thrombolysis and endovascular thrombectomy (EVT), but EVT is not available at all hospitals that offer intravenous thrombolysis. This discrepancy has prompted debate regarding the preferred transport destination. Emergency medical services must decide whether suspected stroke patients should be transported to the nearest stroke center and potentially delay EVT, or to bypass the nearest stroke center in favor of the nearest EVT-capable center, prioritizing the timeliness of EVT over thrombolysis. Quantifying the real-world distance cost of different transport models is essential to understanding their roles in stroke care.

Materials and Methods This cross-sectional study included population data of all census tracts in the contiguous United States from the United States Census Bureau's American Community Survey 2014-2018, stroke centers and EVT-capable centers certified by a national or state organization, and road network data from a public mapping service. Three distances were calculated for each census tract: distance to the nearest stroke center (non-bypass (non-LVO)), distance to the nearest EVT-capable center (bypass), and distance to the nearest stroke center first then transfer to the nearest EVT-capable center 